\title{
Neighborhood Effects and Intimate Partner and Sexual Violence: Latest Results
}

\section{Victoria Frye and Patricia 0'Campo}

In the last two decades, there has been an exponential growth in public health and social scientific research on how the neighborhood physical and social environments impact upon a range of health outcomes. This focus reflects a desire on the part of public health practitioners to once again emphasize the critical role that contextneighborhood and community residential environments-can play in promoting population health. Neighborhood effects on general and youth violence have been extensively studied, and there is a smaller but growing evidence base on the role of the neighborhood environment and the types of violence that often occur "behind closed doors" or among the adults within families: intimate partner and sexual violence.

Intimate partner and sexual violence continue as major public health and social problems worldwide, with an estimated 1 in 5 women and 1 in 10 men victimized in their lifetimes in the United States. ${ }^{1}$ Until recently, most public health and healthcare system efforts to address intimate partner and sexual violence have been healthcare clinic- or agency-based responses to victims of violence. Increasingly, public health practitioners and scientists are developing and testing primary prevention strategies, based in residential neighborhoods and integrating social networks, peer norms, and community mobilization approaches. ${ }^{2,3}$ Despite these efforts and a renewed interest in neighborhood effects research more generally, there is still the need for more qualitative and quantitative research to generate and test strong hypotheses that ultimately will inform intimate partner and sexual violence primary prevention efforts at the neighborhood level. Thus, we developed a call for papers focusing on neighborhood effects on these 2 forms of violence, in an effort to stimulate and identify the most recent work in this critical area. The papers received-a mixture of both qualitative and quantitative reports-offer a sense of where this area of work is moving, as well as locate new areas for further research.

Cunradi and colleagues ${ }^{4}$ use Bayesian space-time models to assess the relationship between neighborhood-level alcohol outlets and rates of intimate partner violence-related calls to police and crime reports. In this ecological study, the authors report associations between off-premise outlets and high rates of partner violence police calls and crime reports, and conclude that further research is needed to elucidate the mechanism by which an alcohol-related area characteristics might relate to individual-level risk of experiencing or perpetrating partner violence.

Rothman and colleagues ${ }^{5}$ focus on whether and how neighborhood-level characteristics are related to partner violence among youth, also called teen dating

Frye is with the Laboratory of Social and Behavioral Sciences of the Lindsley F. Kimball Research Institute of the New York Blood Center, New York, NY, USA; O’Campo is with the Centre for Research on Inner City Health at St. Michael's Hospital of the University of Toronto, Toronto, ON, Canada.

Correspondence: Victoria Frye, Laboratory of Social and Behavioral Sciences of the Lindsley F. Kimball Research Institute of the New York Blood Center, New York, NY, USA. (E-mail: VFrye@NYBloodCenter.org) 
violence. Their study examines neighborhood and teen factors contributing to teen dating violence perpetration among over 1,500 urban, public high school students and reveals that when student reports on neighborhood characteristics are modeled, negative neighborhood-level characteristics are related to increased risk of teen dating violence perpetration; however, when adult data were used, no relations emerged. This inconsistency focuses attention on the important role of perceptions of the neighborhood context and how different population subgroups might differentially perceive, and report on, the same neighborhoods.

Yonas and colleagues ${ }^{6}$ pick up on this theme of the importance of perceptions with a qualitative report on whether and how informal neighborhood leaders believe neighborhood characteristics to be related to intimate partner violence within their neighborhood. Results revealed that informal neighborhood leaders readily linked physical disorder and decay to partner violence perpetration, as well as other socioeconomic conditions of the neighborhood. Importantly, those sampled identified the absence of neighborhood resources and residential capacity to address partner violence as being important determinants for the initiation and maintenance of partner violence in the neighborhood. The findings suggest that the mechanisms of influence that are central to many of the quantitative empirical assessments of the neighborhood environment and intimate partner violence are perceived to be important to partner violence by informal neighborhood leaders.

Also adding to the complex linkages between perceptions of neighborhood conditions and violence perpetration, Reed and colleagues ${ }^{7}$ assess relations among perceived peer and neighborhood social norms around teen dating violence and perpetration of such violence among young, urban men attending community health clinics. They found that the positive relationship between perceived peer norms around dating violence and self-reported perpetration of dating violence diminished in models controlling for the living arrangements of the participants. In fully adjusted models, boys who reported being involved in (general) neighborhood violence and holding traditional gender beliefs were significantly more likely to report perpetrating dating violence.

McDonnell and colleagues ${ }^{8}$ report results of a scale developed specifically to measure women's perceived neighborhood-level social norms around intimate partner violence, a critical first step to measuring social norms at the neighborhood level. The resulting 16-item scale focused on both descriptive and injunctive norms of assistance or intervention into partner violence, and performed well in analyses of internal consistency.

The final paper included in the special section, by Taylor and colleagues, ${ }^{9}$ focuses on the impact of family and the role of perceived norms in their study of attitudes toward corporal punishment among urban parents. Corporal punishment can be conceptualized to exist along a continuum of child maltreatment, a familylevel environmental factor that covaries with intimate partner violence. The paper reports that perceived norms around corporal punishment were strongly associated with positive attitudes toward such punishment. Although focused on another form of control within family relationships, the paper offers evidence that individual-level perceptions of the social environment, in conjunction with individual-level attitudes, may matter to enactment of violence.

Several of the papers in this special issue focus on how perceptions of the neighborhood or social environment potentially relate to either victimization or help-seeking behavior. These analysts and others, typically using qualitative or blended methods, go to the source-communities and/or victims—and in so doing 
interrogate our assumptions about what in the neighborhood environment matters to intimate partner and sexual violence. Some find support for negative effects of social disorganization on violence, with neighborhood residents perceiving that area economic conditions and physical disorder affect partner violence, ${ }^{6}$ whereas others report that female victims of partner violence report fewer neighborly responses to violence than there ought to be, ${ }^{8}$ suggesting that one of the critical mechanisms measured in social disorganization and collective efficacy frameworks, informal social control, may not be strongly at work.

One multilevel report finds that the impact of neighborhood-level characteristics differs for teens, depending upon whose data are aggregated up to the neighborhood level: teens or adults. ${ }^{5}$ Here emerges the issue of whose perceptions of the neighborhood are important, when those perceptions constitute the area-level measures that are modeled as correlates of various forms of partner and sexual violence. Also emerging as important in these collected papers is the role of individuallevel attitudes, albeit in the context of perceived norms around violence, with one report finding that gender beliefs relate to teen dating violence perpetration ${ }^{7}$ and another reporting that perceptions of norms around corporal punishment relate to attitudes toward such behavior. ${ }^{9}$ Finally, the need for illuminating the pathways linking the neighborhood environment and individual-level behavior is raised again in an ecological analysis of alcohol outlet density and partner violence among adults. ${ }^{4}$ Central to this investigative process is the development of appropriate measures specific to neighborhood effects research on intimate partner and sexual violence.

Taken together, these reports remind us that there is much more work to be done in specifying exactly how the neighborhood environment affects experience and perpetration of intimate partner and sexual violence; the research collected in this issue begins to take us down that path. It also reminds us that there is much to be gained by going "back to the drawing board" and using grounded methods to develop new theories, conceptual models, and measures. Such approaches offer hope for linking the lived realities of neighborhood residents, both victims and not, and the group-level social characteristics and processes that may, or may not, be at work to control or facilitate intimate partner and sexual violence.

\section{REFERENCES}

1. Centers for Disease Control and Prevention. Adverse health conditions and health risk behaviors associated with intimate partner violence-United States 2005. MMWR. 2008; 57(5): 113-7.

2. Fujie Parks L, Cohen L, Kravitz-Wirtz N. Poised for prevention: advancing promising approaches for preventing intimate partner violence. The Prevention Institute. 2007. http:// preventioninstitute.org/component/jlibrary/article/id-32/127.html. Accessed January 27, 2011.

3. Davis R, Fujie Parks L, Cohen L. Sexual violence and the spectrum of prevention: towards a community solution. The Prevention Institute. 2006. http://preventioninstitute.org/ component/jlibrary/article/id-97/127.html. Accessed January 27, 2011.

4. Cunradi CB, Mair C, Ponicki W, Remer L. Alcohol outlets, neighborhood characteristics, and intimate partner violence: ecological analysis of a California city. J Urban Health. 2011; 88(1):. doi:10.1007/s11524-011-9549-6.

5. Rothman EF, Johnson RM, Young R, Weinberg J, Azrael D, Molnar BE. NeighborhoodLevel Factors Associated with Physical Dating Violence Perpetration: Results of a Representative Survey Conducted in Boston, MA. J Urban Health. 2011; 88(1):. doi:10.1007/s11524-011-9543-z. 
6. Yonas M, Akers AY, Burke JG, Chang JC, Thomas AL, O'Campo P. Perceptions of prominent neighborhood individuals regarding neighborhood factors and intimate partner violence. J Urban Health. 2011; 88(1):. doi:10.1007/s11524-011-9544-y.

7. Reed E, Silverman JG, Raj A, Decker MR, Miller E. Male perpetration of teen dating violence: associations with neighborhood violence involvement, gender attitudes, and perceived peer and neighborhood norms. J Urban Health. 2011; 88(1):. doi:10.1007/ s11524-011-9545-x.

8. McDonnell KA, Burke JG, Gielen AC, O’Campo P, Weidl M. Women's perceptions of their community's social norms towards assisting women who have experienced intimate partner violence. J Urban Health. 2011; 88(1):. doi:10.1007/s11524-011-9546-9.

9. Taylor CA, Hamvas L, Rice J, Newman DL, DeJong W. Perceived Social Norms, Expectations, and Attitudes toward Corporal Punishment among an Urban Community Sample of Parents. J Urban Health. 2011; 88(1):. doi:10.1007/s11524-011-9548-7. 